\title{
Measurement of the CKM Angle Alpha at the BABAR Detector Using B Meson Decays to Rho Final States
}

\author{
Attila Mihalyi
}




\title{
MEASUREMENT OF THE CKM ANGLE ALPHA AT THE BABAR DETECTOR USING B MESON DECAYS TO RHO FINAL STATES
}

ATTILA MIHALYI

\begin{abstract}
A dissertation submitted in partial fulfillment of the
\end{abstract} requirements for the degree of

DOCTOR OF PHLOSOPHY

(PHYSICS)

at the

UNIVERSITY OF WISCONSIN - MADISON 


\section{Abstract}

This thesis contains the results of an analysis of $B^{0} \rightarrow \rho^{+} \rho^{-}$using 232 million $\Upsilon(4 S) \rightarrow$ $B \bar{B}$ decays collected with the BABAR detector at the $P E P-I I$ asymmetric-energy $B$ Factory at SLAC. From a fitted signal yield of $617 \pm 52$ events, the longitudinal polarizations fraction, $f_{L}$, of the decay is measured to be $0.978 \pm 0.014$ (stat) ${ }_{-0.029}^{+0.021}$ (syst).

The nearly fully longitudinal dominance of the $B^{0} \rightarrow \rho^{+} \rho^{-}$decay allows for a measurement of the time dependent $C P$ parameters $S_{L}$ and $C_{L}$, where the first parameter is sensitive to mixing induced $C P$ violation and the second one to direct $C P$ violation. From the same signal yield, these values are found to be $S_{L}=-0.33 \pm$ 0.24 (stat) $)_{-0.14}^{+0.08}$ (syst) and $C_{L}=-0.03 \pm 0.18$ (stat) \pm 0.09 (syst)

The CKM angle $\alpha$ is then determined, using these results and the branching fractions and polarizations of the decays $B^{0} \rightarrow \rho^{0} \rho^{0}$ and $B^{+} \rightarrow \rho^{+} \rho^{0}$. This measurement is done with an isospin analysis, in which a triangle is constructed from the isospin

amplitudes of these three decay modes. A $\chi^{2}$ expression that includes the measured quantities expressed as the lengths of the sides of the isospin triangles is constructed and minimized to determine a confidence level on $\alpha$. Selecting the solution compatible with the Standard Model, one obtains $\alpha=100^{\circ} \pm 13^{\circ}$. 


\section{Acknowledgments}

I am very grateful for the support and guidance of my adviser Sau Lan Wu, who is always fully committed to helping her students achieve success.

I would also like to thank members of my research group in particular Paul Kutter, Mathew Graham, Mousumi Datta and Jinwei Wu for their friendship and help during my stay at SLAC. In addition I would like to thank Yibin Pan for his assistance when I was starting out at BABAR. This work would not have been possible without the close and fruitful collaboration with Adrian Bevan, Christophe Yeche and Christos

Touramanis. I am forever indebted to them for their hard work and commitment to pursuing this analysis.

I also wish to thank my parents Julianna and Daniel Mihalyi for providing me with great opportunities and constant support to achieve my dreams. Finally I would like to thank my wife Michele, whose love, support and encouragement have been immensely important. 


\section{Contents}

Abstract

Acknowledgments

1 Introduction 1

1.1 Introduction to $\mathrm{CP}$ Violation $\ldots \ldots \ldots \ldots$

1.2 Origins of CP-violation within the $\mathrm{SM} \ldots \ldots \ldots \ldots$

1.3 B-meson Mixing and Time Dependent Formalism . . . . . . . . 10

1.4 Types of $C P$-Violation in the $B$-meson System . . . . . . . . . 14

$1,4.1 C P$-violation in decay $\ldots \ldots \ldots \ldots 14$

1.4 .2 CP-violation in Mixing . . . . . . . . . . 15

1.4.3 CP Violation in Interference Between Decays With and Without

Mixing .................... 16

$1.5 \quad B^{0} \rightarrow \rho^{ \pm} \rho^{\mp}$ Time Dependent Formalism ... . . . . . 17

2 PEP-II $B$ Factory and BABAR Detector 20

2.1 PEP-II Asymmetric B Factory . . . . . . . . . . . . . . . . 22 
2.2 Silicon Vertex Tracker $\ldots \ldots \ldots \ldots \ldots . \ldots . \ldots . \ldots . \ldots$

2.3 Drift Chamber ................... 27

2.4 Detector of Internally Reflected Cherenkov Light . . . . . . . . 32

2.5 Electromagnetic Calorimeter . . . . . . . . . . . . . 36

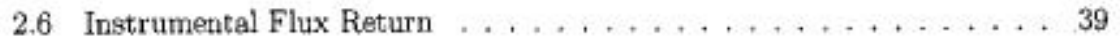

2.7 Trigger ............................ 42

3 Analysis Overview $\quad 45$

3.1 Monte Carlo (MC) Simulation ... . . . . . . . . . . . . 46

3.2 Decay Dỹnamics ... . . . . . . . . . . . . . 48

3.3 Signal Discrimination . . . . . . . . . . . . . . . . 50

3.3.1 Signal and Background Topologies ... . . . . . . . 53

3.4 Vertexing and Vertex Separation . . . . . . . . . . . . . 58

3.5 B-Flavor Tagging $\ldots \ldots \ldots \ldots \ldots 6 . \ldots \ldots$

3.6 Data Sample and Event Reconstruction . . . . . . . . . . . . . 64

3.6.1 Choice of One Candidate per Event ............ 66

3.6 .2 Misreconstructed Signal ... . . . . . . . . . 68

3.7 Maximum Likelihood Fit . . . . . . . . . . . . . . . . . 69

3.8 Probability Density Function Parameterizations . . . . . . . . . 72

3.8.1 Treatment of $\Delta t$ for Signal and Background Events . . . . . . 73

3.8.2 Treatment of correct and wrong track SCF $\Delta t$ resolution . . . . 75

3.9 B-backgrounds ...................... 76 
3.10 Validation of the Fitter . . . . . . . . . . . . . 82

3.10 .1 Toy Monte Carlo Studies . . . . . . . . . . . . . . . . . 83

3.10 .2 Real Monte Carlo Studies . . . . . . . . . . . . 88

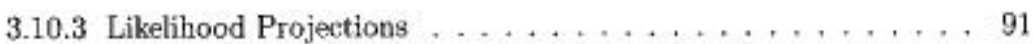

3.11 Fit Results . . . . . . . . . . . . . . . . . . . . . 91

3.12 Evaluation of Systematic Uncertainties ... . . . . . . . 97

3.12 .1 PDF Uncertainties $\ldots \ldots \ldots \ldots \ldots$

3.12 .2 Systematics from the Neural Network . . . . . . . . . . 98

3.12 .3 Systematics from the $B$-background . . . . . . . . 100

3.12 .4 Systematic error from SCE fraction . . . . . . . . . . . . 102

3.12.5 Systematic error from $B$-lifetime and mixing parameters , . . 102

3.12.6 Systematic error from the resolution function, tagging and dilution 103 3.12 .7 Systematic errors from the wrong track SCF . . . . . . . 104

3.12.8 Systematic error from $C P$ violation in transverse signal . . . . 105

3.12 .9 Systematic error from non-resonant events . . . . . . . . 106

$3.12 .10 \mathrm{DCSD}$ decays $\ldots \ldots \ldots \ldots \ldots 7 \ldots \ldots \ldots \ldots$

3.12.10.1 Neglecting interference . . . . . . . . . . 108

3.12 .10 .2 Fit Bias summary $\ldots \ldots \ldots 10 . \ldots$

3.12.10.3 Uncertainty from floating $B$-background yields . . . 110

3.12 .11 SVT Local Alignment . . . . . . . . . . . . . 110

3.12.11.1 Systematic Error Summary . . . . . . . . . 111 
5 Summary

A. Variable Distributions and PDF Shapes

B Correlations Between Discriminating Variables 


\section{List of Figures}

1.1 The rescaled Unitarity Triangle $\ldots \ldots \ldots \ldots$

1.2 Thee and givonic pengtin diagrams contributing to the process $B \rightarrow \rho \rho \quad 18$

2.1 BABAR detector longitudinal cross section. . . . . . . . . . 21

2.2 Schematic view of the SVT ................. 25

2.3 Schematic view of $S V T$ transverse section. . . . . . . . . . 27

2.4 Longitudinal section of the $D C H$ with princspal dimensions . . . . . 29

2.5 Schematic layout of drift cells for the four innermost superlayers . . . 30

2.6 Measurement of $\mathrm{d} E / \mathrm{d} x$ in the $D C H$ as a function of track mamenta . . 31

2.7 Schematics of the DIRC fused silica radiator bar and imaging region. - 33

2.8 Expladed view of the DIRC mechanical support structure. . . . . . . 34

2.9 Kaon-pion separation as a function of track momentum ... . . . . 36

2.10 A longitudinal cross section of the EMC . . . . . . . . . 38

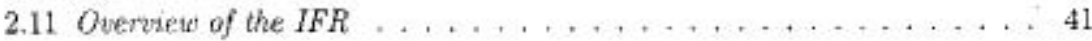

3.1 Comparison of signal and continuum background for $m_{\text {ES }}$ and $\Delta E \ldots 52$

3.2 The input variables tused in training the neural network ... . . . . 56 
3.3 Neural Network efficiencies. . . . . . . . . . . . . . . 57

3.4 Time evolution of the $B-\bar{B}$ system. . . . . . . . . 58

3.5 The PDFs for $m_{\rho}$ and $\rho$ helicity for (top) true and (bottom) fake $a_{1} \pi^{0} .82$

3.6 The $P D F s$ for $m_{\rho}$ and $\rho$ helicity for (top) true and (bottom) fake $a_{1} \pi$. . 83

3.7 The PDFs for $m_{\rho}$ and $\rho$ helicity for (top) true and (bottom) fake $\rho w^{0}$. . 84

3.8 Pull distributions for toy $M C \ldots \ldots \ldots \ldots \ldots$

3.9 Distribution of the likelihood values from the toy MC fits . . . . . . 87

3.10 Comparison of signal and background likelihoods . . . . . . . . . 91

3.11 Comparison of signal, MC and offpeak data likelihoods . . . . . . . 92

3.12 The $m_{\mathrm{ES}}, \Delta E, m_{\pi^{ \pm} \pi^{0}}$ and $\cos \theta_{,}$PDFs projected on data $\ldots . . . .94$

3.13 The PDF for the total likelihood projected on data . . . . . . . 95

3.14 The $\Delta t$ PDF projected on data . . . . . . . . . . . 96

$3.15 M C$ and date comparison of the monomials $L_{0}$ and $L_{2} \ldots \ldots \ldots$. . 99

3.16 NN output before and after the systematic correction . . . . . . . 100

4.1 Isospin Triangles for $B \rightarrow \rho \rho$ longitudinal polarization decays. . . . 115

4.2 Confidence Level on $\alpha$ for a pedagogical example . . . . . . . . . 120

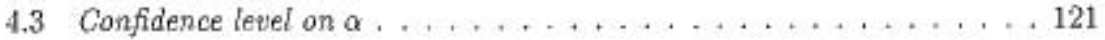

A.1 Distributions and PDFs for $m_{E S}$ and $\Delta E$ for truth matched signal MC 126

A.2 Distributions and PDFs for $m_{E S \text { and }} \triangle E$ for transuersely polarised $S C F 126$ 
A.3 Distributions and PDFs for $m_{E S}$ and $\triangle E$ from longitudinally polarised correct (right) and urong thack (left) SCF ............

A.4 Distributions and PDFs for $m_{\rho}$ and two $\rho$ helicity for truth matched

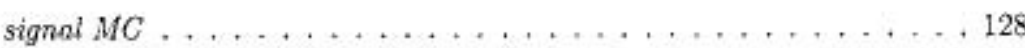

A.5 Distributions and PDFs for $m_{p}$ and $\rho$ helicity for correct(left) and wrong (right) track longitudinally polarized SCF , . . . . . . . 129

A.6 Distributions and PDFs for $m_{\rho}$ and $\rho$ helicity for tronstersly polerized

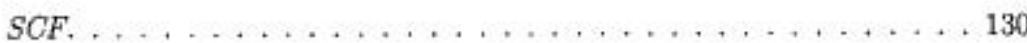

A.7 Distributions and PDFs for Neural net work for true longitudinal signal 131

A.8 Distributions PDFs for Neural net work for true transwerse signal . . 132

A.9 Distributions and PDFs for Neural net work for longitudinal correct track $S C F \ldots \ldots \ldots \ldots \ldots$

A.10 Distribations and PDFs for Neural net work for longitutinal urong track

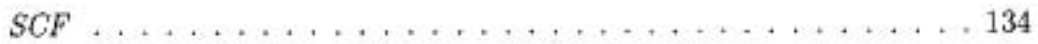
A.11 Distributions and PDFs for Neural net work for transuerse SXF . . . 135

A.12 Distributions and PDFs for continum $\rho$ helicity . . . . . . . 136

A.13 The distributions and PDF for continutm $p$-mass $\ldots \ldots \ldots \ldots 137$

A.14 The variable distributions and PDF shapes for $B^{ \pm} \rightarrow$ ThreeBody. /CLO/138

A.15 The varioble distributions and PDF shapes for $B^{ \pm} \rightarrow$ Four Body. [CL1] 139

A.16 The variable distributions and PDF shapes for $B^{ \pm} \rightarrow$ FiveBody. [CL2] 140 
A.17 The variable distributions and PDF shapes for $B^{ \pm} \rightarrow a_{1}\left(\rightarrow(\rho \pi)^{0}\right) \pi^{ \pm}$.

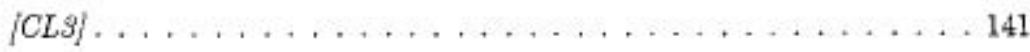

A.18 The variable distributions and PDF shapes for $B^{ \pm} \rightarrow a_{1}\left(\rightarrow(\rho \pi)^{+}\right) \pi^{D}$.

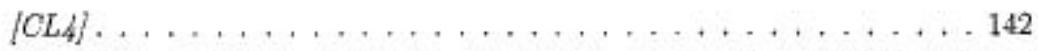

A.19 The variable distributions and PDF shapes for $B^{ \pm} \rightarrow a_{1}\left(\rightarrow(\rho \pi)^{0}\right) \rho^{+}$.

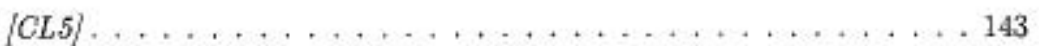

A.20 The variable distributions and PDF shapes for $B^{ \pm} \rightarrow \rho^{+} \rho^{0}$. /CL6/ . . 144

A.21 The variable distributions and PDF shopes for $\left.B^{ \pm} \rightarrow K^{* *} \pi \cdot C L \gamma\right] \ldots 145$

A.22 The variable distributions and PDF shapes for $\left.B^{ \pm} \rightarrow K^{*} \rho, / C L 8\right] \ldots 146$

A.23 The variable distributsons and PDF shapes for $B^{ \pm} \rightarrow c \ldots \ldots . \ldots 147$

A.24 The variable distributions and PDF shapes for $B^{0} \rightarrow$ Four Body. [CL10]148

A.25 The variable distributions and PDF shapes for $B^{0} \rightarrow$ FiveBody. [CL11] 149

A.26 The variable distributions and PDF shapes for $\left.B^{0} \rightarrow \rho \pi . / C L 12\right] \ldots 150$

A.27 The variable distributions and PDF shapes for $B^{0} \rightarrow a_{1} \rho$. [CL13] . . 151

A.28 The variable distributions and PDF shapes for $B^{0} \rightarrow K^{* *} \pi$. [CL14] . . 152

A.29 The variable distributions and PDF shapes for $B^{0} \rightarrow K^{* *} \rho$. /CL15/ . . 153

A.30 The variable distributions and PDF shapes for $B^{0} \rightarrow a_{1}\left(\rightarrow \rho^{0} \pi\right) \pi^{-}$.

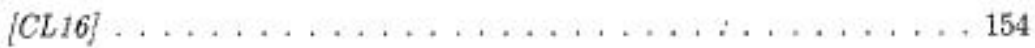

A.31 The variabie distributions and PDF shapes for $B^{0} \rightarrow a_{1}\left(\rightarrow \rho \pi^{0}\right) \pi^{0}$.

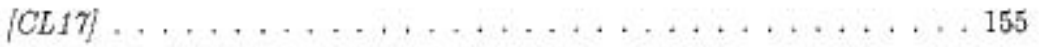

A.32 The variable distributions and PDF shapes for $B^{0} \rightarrow c \ldots \ldots$ 
A.33 $B^{+} \rightarrow c$ (left) and $B^{0} \rightarrow c$ (right) $P D F s$ for $m_{E S}$ parametrised with an

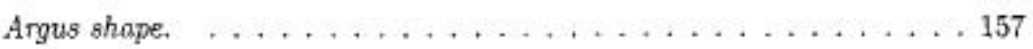

B.1 $2 D$ projection plots of longitudinally polarized truth matched signal MC

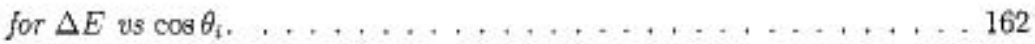

B.2 2D projection plots of longitudinally polarized correct track SCF signal

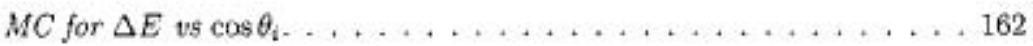

B.3 $2 D$ projection plots of longitudinally polarized unong track SCF signal

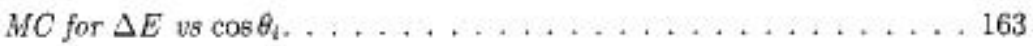

B.4 $2 D$ projection plots of transveresely polarized signal $M C$ for $\Delta E$ vs ous $\theta_{i}, 163$ 


\section{List of Tables}

2.1 PEP-II beam parameters . . . . . . . . . . . . . . 22

2.2 Cross sections, production and trigger rates for the principal physics processes at $10.58 \mathrm{GeV}$ for a luminosity of $3 \times 10^{33} \mathrm{~cm}^{-2} \mathrm{~s}^{-1} \ldots \ldots 43$

3.1 The Monte Carlo samples used in this analysis. . . . . . . . . 47

3.2 Current experimental knowledge of the decay $B \rightarrow \rho \rho \ldots \ldots \ldots \ldots 50$

3.3 The performance of the tagger ............... 63

3.4 Selection efficiencies ..................... 67

3.5 Average multiplicity in Monte Carlo and data. . . . . . . . . 67

3.6 Summary of the fraction of SCF and efficiency for $B^{0} \rightarrow \rho^{+} \rho^{-} \ldots \ldots 6$

3.7 SCF and wrong track fractions, split by tagging categories. . . . . . 71

3.8 The types of PDFs used to model the discriminating variables . . . . 73

3.9 Parameters of the $\Delta t$ model . . . . . . . . . . 75

3.10 Classification of background from charged $B$ 's . . . . . . . 79

3.11 Classification of background from neutral $B^{\prime} s \ldots \ldots \ldots . \ldots 8$

3.12 Results from fits to collections of renl MC samples . . . . . . . . . . 89 
3.13 Average futted polarization for different generated values . . . . . . . 90

3.14 Fit resuits for the final fit to the full onpeak sample ......... 93

3.15 Systematic error summary . . . . . . . . . . . . . . . 112

4.1 Input parameters for the calculation of $\alpha \ldots \ldots \ldots$

B.1 Correlation caefficients for true Longitudinal Signal MC. . . . . . . . 159

B.2 Correlation caefficients for SCF correct track Longitudinal Signal MC. . 159

B.3 Correlation coefficients for SCF wrong track Longitudinal Signal MC. , 159

B.4 Correlation coefficients for onpeak data. . . . . . . . . . 160

B.5 Correlation coefficients for onpeak data. . . . . . . . . . 160

B.6 Correlation coefficients for offpeak data. .............. 160 